\title{
Voluntary rewards mediate the evolution of pool punishment for maintaining public goods in large populations
}

\author{
SUBJECT AREAS: \\ SOCIAL EVOLUTION \\ HUMAN BEHAVIOUR \\ CULTURAL EVOLUTION
}

APPLIED MATHEMATICS

Received

17 October 2014

Accepted

10 February 2015

Published

10 March 2015

Correspondence and requests for materials should be addressed to T.S. (tatsuya.sasaki@ univie.ac.at)

\author{
Tatsuya Sasaki', Satoshi Uchida² \& Xiaojie Chen ${ }^{3}$
}

\begin{abstract}
${ }^{1}$ Faculty of Mathematics, University of Vienna, 1090 Vienna, Austria, ${ }^{2}$ Research Center, RINRI Institute, $101-8385$ Tokyo, Japan, ${ }^{3}$ School of Mathematical Sciences, University of Electronic Science and Technology of China, 611731 Chengdu, China.
\end{abstract}

Punishment is a popular tool when governing commons in situations where free riders would otherwise take over. It is well known that sanctioning systems, such as the police and courts, are costly and thus can suffer from those who free ride on other's efforts to maintain the sanctioning systems (second-order free riders). Previous game-theory studies showed that if populations are very large, pool punishment rarely emerges in public good games, even when participation is optional, because of second-order free riders. Here we show that a matching fund for rewarding cooperation leads to the emergence of pool punishment, despite the presence of second-order free riders. We demonstrate that reward funds can pave the way for a transition from a population of free riders to a population of pool punishers. A key factor in promoting the transition is also to reward those who contribute to pool punishment, yet not abstaining from participation. Reward funds eventually vanish in raising pool punishment, which is sustainable by punishing the second-order free riders. This suggests that considering the interdependence of reward and punishment may help to better understand the origins and transitions of social norms and institutions.

\footnotetext{
C ooperation is costly in the commons dilemma. The evolution of cooperation among nonrelatives with social learning has been a persistent issue approached interdisciplinary, as more than a biological issue ${ }^{1,2}$. Needless to say, those who free ride on the cooperation of others are better off than those who cooperate, unless structural changes are considered in the individual payoff. As is well known, various selective incentives, such as reward, punishment, or ostracism, have been used to modify payoff structures and curb human behaviors $^{3,4}$. Thus far, theoretical and experimental studies have mostly focused on punishment ${ }^{5,6}$, which can sustain a high level of cooperation in providing public goods ${ }^{7-9}$.

The evolution of punishment, however, remains a challenging puzzle ${ }^{6,10-12}$. Punishing is costly. It is thus not an easy task to explore if and how costly punishment pays ${ }^{11}$. Previous studies on the evolution of punishment have also demonstrated that differences in the details of punishment schemes, in particular when a punisher's cost is incurred, can have a large effect ${ }^{13,14}$. One representative type that has been most studied is informal or peer punishment. Peer punishment is inductively modeled, being typically described as: because you wronged me (or someone), I will punish you. As such, peer punishment depends on the assessment of past behaviors ${ }^{15}$.

Continuing costly punishment itself is another public good and thus peer punishment can pave the way for regression to the punishment of free riders through peer punishment (second-order punishment) ${ }^{4,7,16}$. In the same way, third-order punishment and so on are applied. This will result in an infinite regress of costly punishments. Or in situations in which punishment against contributors or retaliation is allowed, these acts can offset the payoff advantage of the existing prosocial punishers over free riders ${ }^{17,18}$. People afraid of antisocial and counter punishments thus might shift the responsibility for sanctioning to others ${ }^{19}$.

Along this line of thinking, we turn to another representative type of punishment, formal or pool punishment. Pool punishment is a "preemption" system that is set in place before forming joint enterprises (i.e., a public good game) and without knowing if there is a free rider among the participants, and subsequently each participant is offered the opportunity to contribute to a fund for pool punishment ${ }^{12,14,20-25}$. Recent studies show that considering second-order punishment results in pool punishment becoming more effective than peer punishment for stabilizing a cooperative state and participants are more likely to prefer pool punishment over peer punishment ${ }^{14,23,26,27}$. In pool punishment, it is assumed that a centralized authority, once established, can exclusively control the incentives, so that it suppresses non-responsible punishment and excludes the possibility of free riders higher than second-order.
} 
Apart from the issue of system stabilization, there still remains another issue relevant for the evolution of costly punishment: the emergence problem. Indeed, punishing right and left in large populations of free riders will require considerable effort and expense for pool punishers. Reflecting this, it is often explicitly assumed that pool punishment becomes active if at least a threshold number of players, more than one, contribute to $\mathrm{it}^{28-30}$. This means that in such large populations it is not easy to successfully start up costly punishment ${ }^{31,32}$, even with considering punishment of second-order free riders ${ }^{33}$ (Fig. 1a,b).

For the last decade, several attempts have tried to resolve the emergence problem. Most of the theoretical results have been based on assuming small, finite populations and analyzing those stochastic dynamics ${ }^{13,24,34-36}$. In addition, optional participation and mutual aid games (MAGs) have been considered as key factors in a resolution ${ }^{14,22,25}$. When participation in games is optional, players can simply escape a social trap of mutual defection ${ }^{37,38}$. MAGs are variants of public good games (PGGs). In PGGs the resulting benefits are shared equally among all members in the group. In MAGs it is not allowed to benefit from one's own contribution to the public goods provision ${ }^{13,20}$. That is, MAGs deal with excludable goods, not public goods, and combined with optional participation, are also two-fold exclusion. As such, previous studies have shed light on excludable good games in small populations.

Here we turn to pool reward ${ }^{39,40}$, thereby we tackle the emergence of pool punishment in non-excludable good games in very large populations. We model a situation like a matching fund that usually arises for charity or common goods, in which contributors donate to a nonprofit source outside. Then the external source, enhancing the input, will make returns to a broader range of beneficiaries that include the contributors. Previous studies have investigated reward and punishment, often comparatively ${ }^{41-45}$, and have also examined the selection or interplay of these incentives ${ }^{46-52}$. It is thus surprising that little is known about what happens if those who commit to pool punishment are promptly rewarded, rather than through iterated interactions or reputation.

Rewarding is costly. The pool reward being considered allows for receiving self-returns from one's own contribution as well as sharing in other's contribution without contributing, similar to PGGs. It follows that a pool reward can suffer from those who take a free ride on the reward-fund raising. From the viewpoint of its initiator, rather than punishing, rewarding can be less expensive and thus more efficiently stimulate cooperative behaviors ${ }^{4,43,46,52}$. Indeed, voluntary rewarding can be maintained even in public good games with second-order free riders $^{39,53}$. It is thus predicted that a pool reward that also rewards volunteers to pool punishment will provide a foothold for the initially rare volunteers to proliferate, overcoming the emergence problem even without the assistance of the optional participation. We shall confirm this prediction by using the following game-theoretical model.

\section{Methods}

Evolutionary games for a public good and multi-strategy interactions. We consider a well-mixed, infinitely large population. We assume that a player is more likely to adopt other player's strategy earning a higher payoff ("imitate better"). In the population this can be implemented by considering replicator dynamics ${ }^{54,55}$. We analyze the replicator dynamics for five strategies that consist of four types of participants in the PGGs: (i) cooperators (C) contribute to the PGG, but not to the incentive funds; (ii) defectors (D) do not contribute at all; (iii) punishers $(\mathrm{P})$ contribute to the PGG and to the punishment fund; (iv) rewarders contribute to the PGG and to the reward fund (R); and (v) nonparticipants $(\mathrm{N})$. We denote as $x_{S}$ and $P_{S}$ the relative frequency and expected payoff for each strategy $S=\mathrm{C}, \mathrm{D}, \mathrm{P}, \mathrm{R}$, or $\mathrm{N}$ (thus, $0 \leq x_{S} \leq 1$ and $\sum_{S} x_{S}=1$ ). The replicator dynamics for the five strategies are given by $\dot{x}_{S}=x_{S}\left(P_{S}-\bar{P}\right)$, in which $\bar{P}$ describes the average payoff over the population, that is $\bar{P}=\sum_{S} x_{S} P_{S}$.

Game procedure and parameters. A group for the public good interaction consists of $n$ $>1$ members who are randomly chosen from the population. First, each of the members is offered an opportunity to participate in the PGG. If participating, then each participant will be subsequently offered distinct opportunities to contribute, to the reward, then the punishment, and finally PGG. Each contribution to the PGG, reward or punishment fund means an investment of $c_{1}, c_{2}, c_{3}>0$, respectively, at a cost to the contributor itself. In the PGG, the resulting benefits, multiplied by factors $r_{1}>1$, are equally shared by all participants, excluding $\mathrm{N}$-players. To examine a previous, problematic situation in which $\mathrm{C}, \mathrm{D}$, and $\mathrm{N}$ coexist, in particular we assume that $2<r_{1}<n^{37}$. In the reward fund, the resulting rewards, multiplied by intermediate factors $r_{2}$ with $1<r_{2}<n$, is shared, yet not always equally, among all of the contributors to the PGG (C-, R-, and P-players), excluding D- and $\mathrm{N}$-players ${ }^{39,40,43}$. We assume weights $k_{\mathrm{RP}}, k_{\mathrm{RR}} \geq 0$ for the $\mathrm{P}$ - and Rplayers' share. In the punishment fund, non-contributors to the punishment (D-, C-, and R-players) incur fines. We assume that the fines are proportional to the contribution accumulated over all P-players ${ }^{14,20}$, with proportionality factor $r_{3}>1$ and weights $k_{\mathrm{PC}}, k_{\mathrm{PR}}$ $\geq 0$ for the $\mathrm{C}$-and R-players' fines. Finally, the fifth type (v) non-participant is a loner that independently earns a small payoff $g>0$. Hence, we have the individual payoff for an interaction, $f_{S}$, of each strategy $S=\mathrm{C}, \mathrm{D}, \mathrm{P}, \mathrm{R}$, or $\mathrm{N}$, as follows:

$$
\begin{aligned}
& f_{C}=\frac{b_{1}\left(n_{C}+n_{R}+n_{P}+1\right)}{n-n_{N}}-c_{1}+\frac{b_{2} n_{R}}{\left(n_{C}+1\right)+k_{R R} n_{R}+k_{R P} n_{P}}-k_{P C} b_{3} n_{P}, \\
& f_{D}=\frac{b_{1}\left(n_{C}+n_{R}+n_{P}\right)}{n-n_{N}}-b_{3} n_{P}, \\
& f_{P}=\frac{b_{1}\left(n_{C}+n_{R}+n_{P}+1\right)}{n-n_{N}}-c_{1}+\frac{k_{R P} b_{2} n_{R}}{n_{C}+k_{R R} n_{R}+k_{R P}\left(n_{P}+1\right)}-c_{3}, \\
& f_{R}=\frac{b_{1}\left(n_{C}+n_{R}+n_{P}+1\right)}{n-n_{N}}-c_{1}+\frac{k_{R R} b_{2}\left(n_{R}+1\right)}{n_{C}+k_{R R}\left(n_{R}+1\right)+k_{R P} n_{P}}-c_{2}-k_{P R} b_{3} n_{P}, \\
& f_{N}=g,
\end{aligned}
$$

No reward Compulsory participation
No reward Optional participation
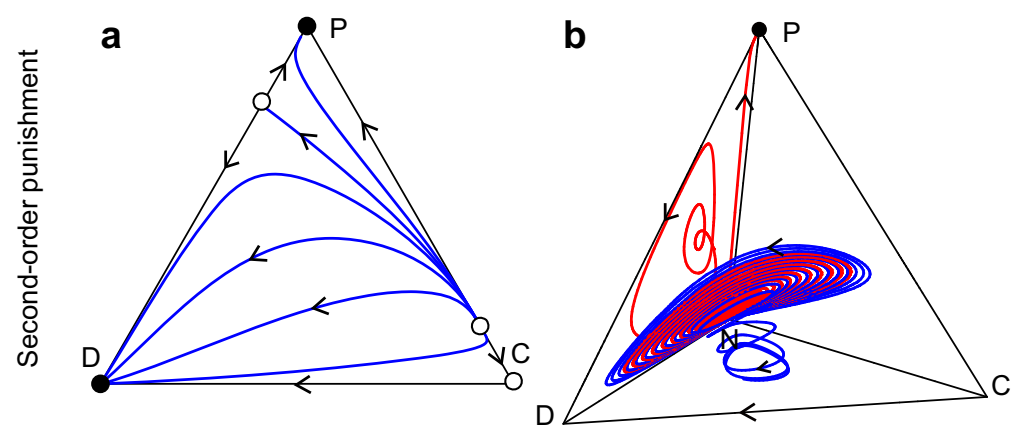

Reward Compulsory participation

\section{D}

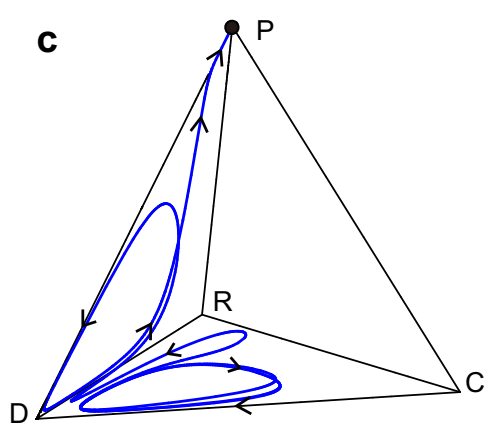

Figure 1 Evolution of pool punishment. With no reward, (a) bistability of states with all P-players (P node) or all D-players (D node) for compulsory participation or (b) bistability of the $\mathrm{P}$ node or periodic oscillations among $\mathrm{C}, \mathrm{D}$, and $\mathrm{N}$ for optional participation. (c) Replace non-participant $\mathrm{N}$ with R. As in panel b, on the CDR face the population states oscillate along periodic closed orbits. In contrast to panels a and b, rare P-players, rewarded, can invade to the CDR face. Typically, the population state will converge to the DPR face, on which the dynamics is repelling. The trajectory then will come close to the edges connecting the three nodes D, P, and R, and finally attain the P node. Parameter values are: $n=5, c_{1}=1, r_{1}=3, c_{2}=1, r_{2}=2, c_{3}=0.1, r_{3}$ $=1.6, k_{\mathrm{RP}}=2, k_{\mathrm{PC}}=1$, and $g=1$. The system includes second-order punishment. Open and filled circles denote, respectively, unstable and asymptotically stable equilibria. 
in which $n_{S}$ denotes the number of $S$-player among $(n-1)$ co-players, $b_{1}=r_{1} c_{1}, b_{2}=$ $r_{2} c_{2}$, and $b_{3}=r_{3} c_{3}$. The expected payoff for each strategy is given by

$P_{S}=\sum_{\substack{n_{C}+n_{D}+n_{P}+n_{R}+n_{N}=n-1 \\ 0 \leq n_{C}, n_{D}, n_{P}, n_{R}, n_{N} \leq n-1}} \frac{(n-1) !}{n_{C} ! n_{D} ! n_{P} ! n_{R} ! n_{N} !} x_{C}^{n_{C}} x_{D}^{n_{D}} x_{P}^{n_{P}} x_{R}^{n_{R}} x_{N}^{n_{N}} f_{S}$, in which $\frac{(n-1) !}{n_{C} ! n_{D} ! n_{P} ! n_{R} ! n_{N} !} x_{C}^{n_{C}} x_{D}^{n_{D}} x_{P}^{n_{P}} x_{R}^{n_{R}} x_{N}^{n_{N}}$ describes the probability of finding the specific

$(n-1)$ co-players which includes $n_{S} S$-players $(S=\mathrm{C}, \mathrm{D}, \mathrm{P}, \mathrm{R}$, and $\mathrm{N})$.

Here, it has been assumed that there are participants of more than one, and if a participant is single, she or he acts as a non-participant and earns the same payoff $g^{31,37}$. In the model we consider that the reward weight $k_{\mathrm{RP}}$ and $k_{\mathrm{RR}}$ describe an extra bonus for the one who contributed not only the PGG but also another public fund. Thus, $k_{\mathrm{RP}}$ and $k_{\mathrm{RR}}$ are supposed to be greater than 1 . In the punishment weights, $k_{\mathrm{PC}}$ and $k_{\mathrm{PR}}$ are usually smaller than 1, denoting a discount factor for the one who did the second-order but not first-order free riding. For simplicity, we hereafter assume that $k_{\mathrm{RR}}-1$ and $k_{\mathrm{PR}}$ offset each other and in particular $k_{\mathrm{RR}}=1$ and $k_{\mathrm{PR}}=0$.

\section{Results}

We, in terms of evolutionary game theory ${ }^{54}$, show that voluntary rewarding for pool punishers can lead to a state in which all are Pplayers, no matter whether participation is compulsory or optional.

Stability of a coercive society. We start with analyzing local stability of the all-P state. In particular for the all-P state to be robust for the invasion of a rare C-player, we consider second-order punishment with $k_{\mathrm{PC}} r_{3}>1 /(n-1)$, under which there is no temptation to switch to $C$ when all play $P$, unless specifically stated otherwise. It is not difficult to also know from equation (1) under which conditions the all-P state is stable against the invasion of a rare $\mathrm{D}$ - or $\mathrm{N}$-player. In the case of $\mathrm{D}$ this is when $c_{1}\left(1-r_{1} / n\right)<c_{3}\left[(n-1) r_{3}-1\right]$ holds, where the left and right sides describe the marginal costs for cooperating in PGGs and for being punished by $n-1$ punishers, respectively. In the case of $\mathrm{N}$ the condition is that $g<c_{1}\left(r_{1}-1\right)-c_{3}$, where the right side means the payoff for the group of all P-players.

Conditions of rock-scissors-paper cycles. It is known that there can exist two kinds of periodic cycles among three strategies. It is clear that the last inequality above is also a sufficient condition that $\mathrm{C}$ dominates $\mathrm{N}$. Considering also that $\mathrm{N}$ dominates $\mathrm{D}$ with $g>0$ and that $\mathrm{D}$ dominates $\mathrm{C}$ with $r_{1}<n$, it follows that when the PGG multiplication factor $r_{1}$ is greater than 2, C-, D-, and N-players alternatively become dominant in the population ${ }^{37,38}$. Otherwise, the population which consists of the three strategies will end up with a homogeneous state in which all play $\mathrm{N}^{37}$. We thus focus on PGGs with $r_{1}>2$ (and thus $n>2$ ) in what follows. In addition, to hold such periodic oscillations among another triplet C-, D-, and Rplayers, it is necessary that $c_{1}\left(1-r_{1} / n\right)<c_{2}\left(r_{2}-1\right)$ holds ${ }^{39}$. Based on these rock-scissors-paper-type cycles, we shall investigate the evolutionary dynamics for more than three strategies.

With no reward, pool punishment never emerges (Fig. 1a,b). We first consider combinations of C-, D-, P-players with or without $\mathrm{N}$-players. We show that no P-players evolve if they are initially very rare, whatever the condition of participation. Let us start by compulsory participation (Fig. 1a). In a population which exclusively consists of $\mathrm{P}$ and $\mathrm{D}$ (or $\mathrm{C}$ ), the replicator dynamics exhibit a bi-stable system: depending on the initial fraction of Pplayers in the population, the population can evolve either to a state of all P-players or a state of all D-players (or all C-players). By assumption D-players are always better off than C-players. Thus, for the three strategies, the dynamics exhibit bistability of the two homogeneous states for P-players or D-players (all-P state and all-D state). Next is in the case of optional participation (Fig. 1b). In competition among three strategies $\mathrm{C}, \mathrm{D}$, and $\mathrm{N}$, it is supposed that the CDN face is filled with periodic closed orbits surrounding a center ${ }^{37}$ (see Supplementary Fig. S1 for detailed phase portraits on the faces). For a coexisting state of $C, D$, and $N$ within the $C D N$ face, a rare, innovative $\mathrm{P}$-player cannot invade, because the time average of the transversal growth rate (i.e., difference of the expected payoff of a rare P-payer and the average payoff over the population) for the rare $\mathrm{P}$-player is negative per punishing $\operatorname{cost} c_{3}$, which is the same as in the case of peer punishment ${ }^{31}$. Thus, in the given parameter settings, the dynamics exhibit bistability of the all-P state and periodic oscillations among C, D, and N (see Supplementary Fig. S2 for time series).

With reward, pool punishment emerges for compulsory participation (Figs. 1c and 2a). Replacing non-participation with a pool reward only leads to the similar dynamics on the corresponding CDR face, which is filled with periodic closed orbits surrounding a center ${ }^{39}$ (Supplementary Fig. S1). It is obvious that the dynamics on the CDP face are unchanged. With an extra reward for $\mathrm{P}$-players with $k_{\mathrm{RP}}>1$, the even rare P-player can be encouraged to
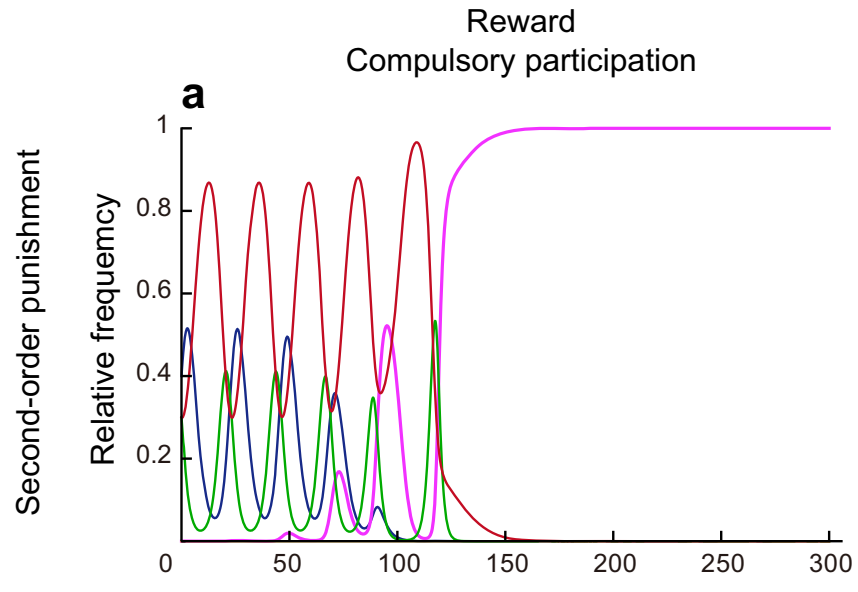

Cooperators

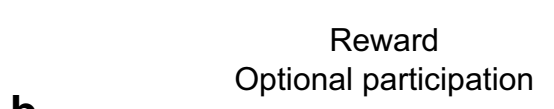

b

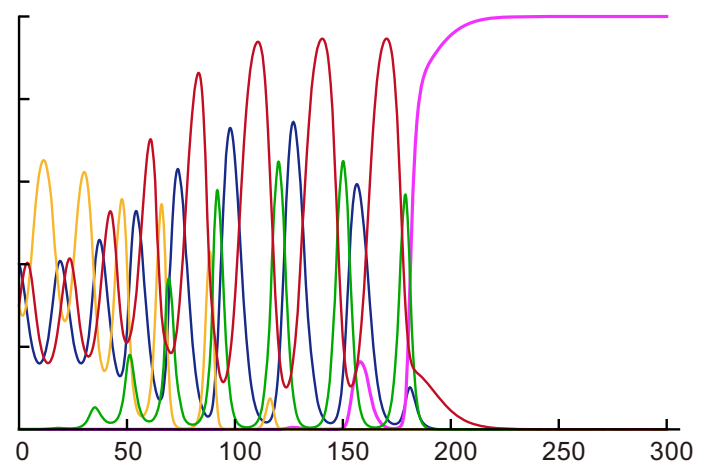

Time

Figure 2 Via reward to punishment. Time series of the frequencies of five strategies C (blue), D (red), P (purple), R (green), and N (yellow). (a)

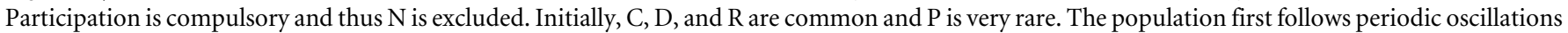

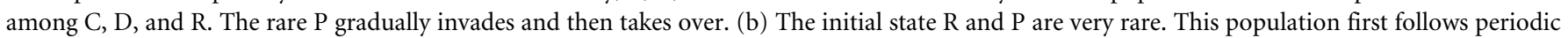

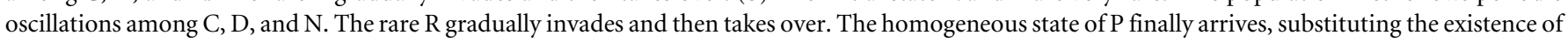

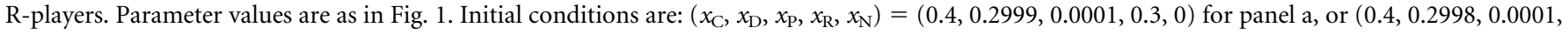
$0.0001,0.3)$ for panel $b$. The system includes second-order punishment. 
invade the coexisting population on the CDR face. Numerical simulations show that the population state will typically come close to the DPR face, increasing in the fraction of P-players and decreasing in that of C-players. This is because of second-order punishment. Among the three strategies of $\mathrm{D}, \mathrm{P}$, and $\mathrm{R}$, the dynamics are repelling (Supplementary Fig. S1). As time goes by, the trajectories of population states will converge to the boundaries connecting the three homogenous states for D, P, and R. Considering that P-players are better off than R-players and R-players are better off than D-players, it is understood that the trajectories will be attracted to the all-P state, which again is robust for invasions of rare $\mathrm{D}$ - or C-players.

Pool reward emerges for optional participation (Fig. 3). To expand the applicable range of pool-reward, we also consider a case where participation is optional. It turns out that with sufficiently high degrees of the reward multiplication factor $r_{2}$, rare R-players can invade the $\mathrm{CDN}$ face, replacing $\mathrm{N}$-players. The population state will eventually be attracted to a periodic orbit on the DNR face (see Supplementary Fig. S1 for detailed phase portraits on the faces). We remark that despite the fact that C-players exploit rewards by R-players, R-players can sprout in the presence of these second-order free riders. The successful invasion of a rare R-player deserves an example of the well-known Simpson's paradox ${ }^{37,56,57}$ for second-order social dilemmas: in spite of the burden of costs for rewarding in each game, the rare R-player's payoff, when it is averaged over the whole population, will be better than the second-order free rider C-player's payoff ${ }^{13,58}$. This is in striking contrast to the former case in pool punishment (Fig. 1b). The DNR face, shared in Fig. 1c, is an "interface" to connect to the evolution of pool punishment and thus opens the door to the full course of the five strategies, as in what follows.

With reward, pool punishment emerges for optional participation (Fig. 2b). The initial state of the population almost exclusively consists of C-, D-, and N-players, and R- and P-players are given only at very small rates. The population first follows periodic oscillations among the resident three strategies. Similar to the last case, the initially rare R-players then start to gradually spread in the population, replacing N-players. The R-players then can take over almost all of the population. However, the all-P state finally arrives, substituting R-players. Without the intermediate sequence of a rise and fall of voluntary rewarding, we can only have continuous oscillations among $\mathrm{C}, \mathrm{D}$, and $\mathrm{N}$.

With no second-order punishment, pool punishment is unstable (Supplementary Figs. S3 and S4). We explore effects of no punishment of C-players, who do not shoulder the punishment fee $c_{3}>0$. It is obvious that with no second-order punishment, $\mathrm{C}$ dominates $\mathrm{P}$ : switching the strategy from $\mathrm{P}$ to $\mathrm{C}$ is beneficial, whatever others do (see equation (1) with $k_{\mathrm{PC}}=0$ ). Therefore, the all-P state is unstable against the C's invasion and a small random shock will cause that a population of $\mathrm{P}$-players will converge to a boundary state that completely excludes P-players. For compulsory participation with no reward, thus such a population will be eventually attracted by the all-D state (Supplementary Fig. S3a), and for optional participation with no reward, by cycles among $\mathrm{C}$, $\mathrm{D}$, and N (Supplementary Fig. S3b). With pool reward, however, the bonus weight for P-players $k_{\mathrm{RP}}>1$ can lead populations to temporally increase in P-players. The trajectories of population states then can converge to heteroclinic cycles, among C, D, R, and $P$ for compulsory participation (Supplementary Figs. S3c and S4a). In particular, for optional participation the population will stay at an almost-all-N state for a long time on a heteroclinic cycle connecting the five homogeneous states for C, D, N, R, and P (Supplementary Fig. S4b).

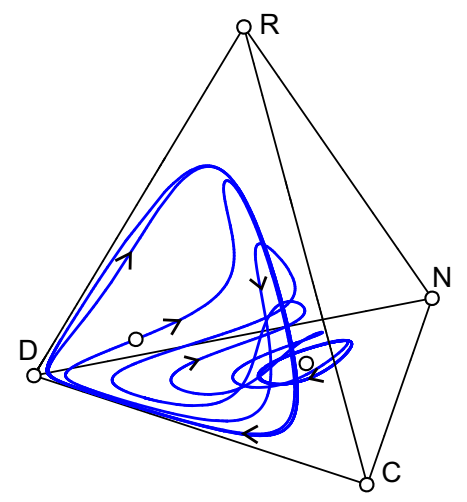

Figure 3 | Via withdrawal to reward. Participation is optional. Initially, C, $\mathrm{D}$, and $\mathrm{N}$ are common and $\mathrm{R}$ is very rare. The population first follows periodic oscillations among $\mathrm{C}, \mathrm{D}$, and $\mathrm{N}$. The rare $\mathrm{R}$ gradually invades the population, substituting the existence of $\mathrm{N}$-players. The dynamics shift to periodic oscillations among C, D, and R. Parameter values are as in Fig. 1.

We examine our main results for a certain range of the model parameters and initial conditions. Our main results that reward funds facilitate the emergence of costly pool punishment are robust against the various initial conditions, whether participation is optional or not (Supplementary Fig. S5). In particular we numerically explore the lower bound of the reward weight for punishers $k_{\mathrm{RP}}$, with various settings of other parameters, $r_{1}, r_{2}, c_{2}$, and $c_{3}$ (Figs. $4 \mathrm{~b}, \mathrm{~d}$ and Supplementary Fig. S6). In Figs. $1 \mathrm{c}$ and $2 \mathrm{~b}$ we also investigate effects of (i) different group sizes $n$, (ii) different combinations of multiplication factors in PGGs and reward funds, $r_{1}$ and $r_{2}$ (Fig. 4a,c), (iii) nonlinearity of benefit production functions (Supplementary Text S1 and Fig. S7), and (iv) pool punishment which imposes constant fees. None of the variants (i)-(iv) qualitatively affect the main results.

\section{Discussion}

Carrot or stick? This is a commonly used dichotomy in studies on selective incentives. Here we have focused on interdependence of reward and punishment. The evolution of costly punishment indeed will be promoted provided ample positive incentives that covers its net cost. In the case preferring costly punishment is a rational behavior. Thus, the core problem has been whether efforts to provide such rewards can endogenously evolve. Only a few studies have explored the evolution of a meta-norm that rewards peer punishers ${ }^{59-62}$. We have instead considered pool reward in $n$-person public good games, which can proliferate when rare even in the presence of second-order free riders. We examined a mediation effect of pool reward on overcoming the emergence problem of pool punishment. It turned out that considering pool reward leads to completing an evolutionary transition of societies in different equilibrium states, with norm deviators or norm followers. The latter state is protected by pool punishment.

Looking back to the real world, a law for an official subsidy or tax reduction to smoothly promote social changes (e.g., green cars and eco-friendly home) often includes its own expiration conditions. In our model, with achievement of a foothold for the evolution of pool punishment, the pool reward becomes evolutionarily retired. These mediation dynamics can be seen for variants of the model. For instance, rewarding mediation is applicable to nonlinear public good games in which the benefit production function has decreasing returns to scale ${ }^{32}$. This is also in threshold public good games in which a certain level of cooperation is required for producing public goods ${ }^{40,63}$. In either case, considering a sufficiently concave benefit function, the homogeneous state for cooperators turns into a stable state and even punishing free riders is redundant to maintain cooperation. 
a

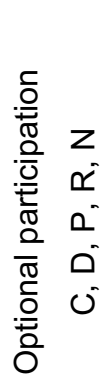

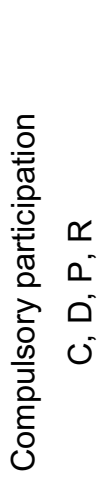

C
4

3
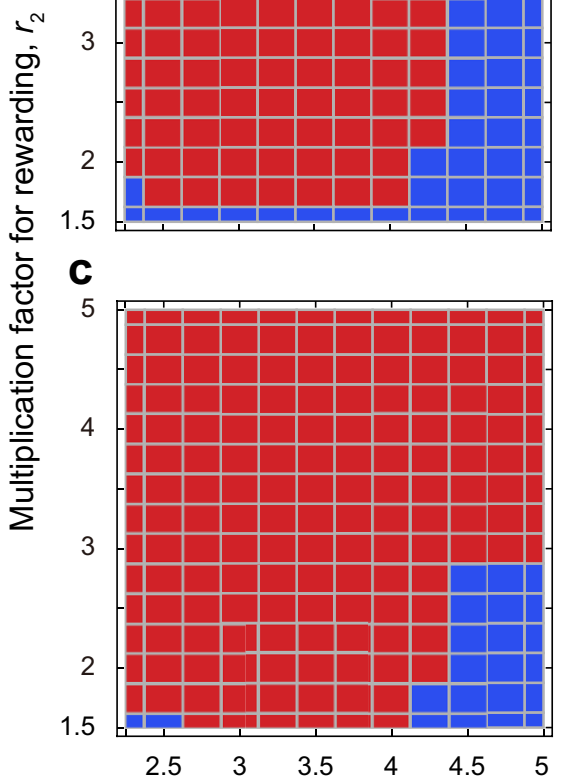

\section{b}

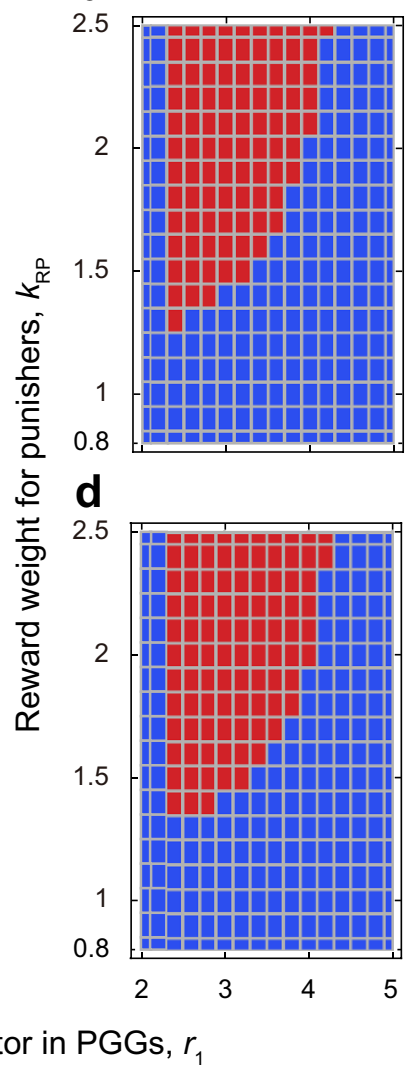

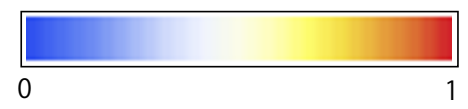

Final frequency of punishers

Figure $4 \mid$ Effects of reward cost and weight on the evolution of pool punishment. Initial conditions are: $\left(x_{\mathrm{C}}, x_{\mathrm{D}}, x_{\mathrm{P}}, x_{\mathrm{R}}, x_{\mathrm{N}}\right)=(0.33,0.338,0.001,0.001$, $0.33)$ for panels a and $\mathrm{b}$, and $\left(x_{\mathrm{C}}, x_{\mathrm{D}}, x_{\mathrm{P}}, x_{\mathrm{R}}\right)=(0.33,0.339,0.001,0.33)$ for panels $\mathrm{c}$ and $\mathrm{d}$. Other parameter values are as in Fig. 1.

The essence of sustaining pool punishment is its prior commitment scheme followed by second-order punishment. Exploring if and how such a commitment system can emerge is out of the range of the model considered. Second-order punishment has been found to effectively prevent second-order free riders from eroding the voluntary sanctioning system ${ }^{7,64,65}$. In the case of peer punishment, it has also been reported that second-order punishment is not likely to be observed $^{62}$. In contrast to this, pool punishment of second-order free riders is often conspicuously observed (i.e., against tax evaders). However, each individual is not supposed to transcendentally abide by the norm of pool punishment. In particular, in the very beginning when people never had concepts of pool punishment and thus there are also second-order free riders, how does a norm that assesses second-order free riders as bad emerge ${ }^{66} \mathrm{~A}$ better understanding of this could be relevant to the quest to understand the "roots of sanctioning institutions" ${ }^{\prime 23}$. As such, the fascinating origin of norm assessment for second-order pool punishment deserves further investigation.

Nowadays, various modern issues of commons, such as energy, natural environment, and climate change, are reaching every corner and covering all stages of human lives. As such, it appears that there is almost no time or space for people to opt out of both the corresponding dilemma situations and the related laws ${ }^{34,67}$. Results, based on compulsory participation but voluntary rewards, thus could be more applicable than previous theories with optional participation ${ }^{14,34}$. This implies an improved scenario to accomplish Garrett Hardin's recipe for the commons: mutual coercion mutually agreed upon ${ }^{1}$. In
Isaiah Berlin's concept ${ }^{68}$, optional participation (with "leaving loners alone"36) can be viewed as a negative liberty, freedom from interference in individual payoff by other players.

In contrast to this, voluntary rewards could be a positive liberty, freedom aimed at modifying the payoff of others. Recent studies have also shown that participants who enable an effect on one another through a majority vote prefer a coercive society with second-order pool punishment ${ }^{27}$. We have revealed that in a broad range of conditions with large populations, non-excludable public goods, or general benefit functions, only having optional participation is often not sufficient ${ }^{32,67}$, but when combined with voluntary rewards, can be effective for establishing pool punishment. All in all, the results may suggest: through positive liberty, corrective coercion.

1. Hardin, G. The tragedy of the commons. Science 162, 1243-1248 (1968).

2. Ostrom, E. Governing the Commons: The Evolution of Institutions for Collective Action. (Cambridge Univ Press, Cambridge, UK, 1990).

3. Olson, M. The Logic of Collective Action: Public Goods and the Theory of Groups. (Harvard Univ Press, Cambridge, MA, 1965).

4. Oliver, P. Rewards and punishments as selective incentives for collective action: theoretical investigations. Am. J. Sociol. 85, 1356-1375 (1980).

5. Balliet, D., Mulder, L. B. \& Van Lange, P. A. Reward, punishment, and cooperation: a meta-analysis. Psychol. Bull. 137, 594-615 (2011).

6. Guala, F. Reciprocity: weak or strong? What punishment experiments do (and do not) demonstrate. Behav. Brain. Sci. 35, 1-15 (2012).

7. Axelrod, R. An evolutionary approach to norms. Am. Polit. Sci. Rev. 80, 1095-1111 (1986).

8. Fehr, E. \& Gächter, S. Altruistic punishment in humans. Nature 415, 137-140 (2002). 
9. Mathew, S. \& Boyd, R. Punishment sustains large-scale cooperation in prestate warfare. Proc. Natl Acad. Sci. USA 108, 11375-11380 (2011).

10. Colman, A. M. The puzzle of cooperation. Nature 440, 744-745 (2006).

11. Milinski, M. \& Rockenbach, B. Human behaviour: Punisher pays. Nature 452, 297-298 (2008).

12. Kosfeld, M., Okada, A. \& Riedl, A. Institution formation in public goods games. Am. Econ. Rev. 99, 1335-1355 (2009).

13. De Silva, H., Hauert, C., Traulsen, A. \& Sigmund, K. Freedom, enforcement, and the social dilemma of strong altruism. J. Evol. Econ. 20, 203-217 (2010).

14. Sigmund, K., De Silva, H., Traulsen, A. \& Hauert, C. Social learning promotes institutions for governing the commons. Nature 466, 861-863 (2010).

15. Nowak, M. A. Evolving cooperation. J. Theor. Biol. 299, 1-8 (2012).

16. Boyd, R. \& Richerson, P. J. Punishment allows the evolution of cooperation (or anything else) in sizable groups. Ethol. Sociobiol. 13, 171-195 (1992).

17. Nikiforakis, N. Punishment and counter-punishment in public good games: Can we really govern ourselves? J. Public Econ. 92, 91-112 (2008).

18. Rand, D. G. \& Nowak, M. A. The evolution of antisocial punishment in optional public goods games. Nat. Commun. 2, 434 (2011).

19. Bartling, B. \& Fischbacher, U. Shifting the blame: on delegation and responsibility. Rev. Econ. Stud. 79, 67-87 (2012).

20. Yamagishi, T. The provision of a sanctioning system as a public good. J. Pers. Soc. Psychol. 51, 110-116 (1986).

21. Andreoni, J. \& Gee, L. K. Gun for hire: delegated enforcement and peer punishment in public goods provision. J. Public. Econ. 96, 1036-1046 (2012).

22. Bodnar, T. \& Salathé, M. Governing the global commons with local institutions. PLoS ONE 7, e34051 (2012).

23. Zhang, B., Li, C., De Silva, H., Bednarik, P. \& Sigmund, K. The evolution of sanctioning institutions: an experimental approach to the social contract. Exper. Econ. 17, 285-303 (2014).

24. Abdallah, S. et al. Corruption drives the emergence of civil society. J. R. Soc. Interface 11, 20131044 (2014).

25. Schoenmakers, S., Hilbe, C., Blasius, B. \& Traulsen, A. Sanctions as honest signals - the evolution of pool punishment by public sanctioning institutions. J. Theor. Biol. 356, 36-46 (2014).

26. Traulsen, A., Röhl, T. \& Milinski, M. An economic experiment reveals that humans prefer pool punishment to maintain the commons. Proc. Bio. Sci. 279, 3716-3721 (2012).

27. Hilbe, C., Traulsen, A., Röhl, T. \& Milinski, M. Democratic decisions establish stable authorities that overcome the paradox of second-order punishment. Proc. Natl Acad. Sci. USA 111, 752-756 (2014).

28. Boyd, R., Gintis, H. \& Bowles, S. Coordinated punishment of defectors sustains cooperation and can proliferate when rare. Science 328, 617-620 (2010).

29. Raihani, N. J. \& Bshary, R. The evolution of punishment in $n$-player public goods games: a volunteer's dilemma. Evolution 65, 2725-2728 (2011).

30. Vasconcelos, V. V., Santos, F. C. \& Pacheco, J. M. A bottom-up institutional approach to cooperative governance of risky commons. Nat. Clim. Chang. 3 , 797-801 (2013).

31. Brandt, H., Hauert, C. \& Sigmund, K. Punishing and abstaining for public goods. Proc. Natl Acad. Sci. USA 103, 495-497 (2006).

32. Mathew, S. \& Boyd, R. When does optional participation allow the evolution of cooperation? Proc. Bio. Sci. 276, 1167-1174 (2009).

33. Dercole, F., De Carli, M., Della Rossa, F. \& Papadopoulos, A. V. Overpunishing is not necessary to fix cooperation in voluntary public goods games. J. Theor. Biol. 326, 70-81 (2013).

34. Hauert, C., Traulsen, A., Brandt, H., Nowak, M. A. \& Sigmund, K. Via freedom to coercion: the emergence of costly punishment. Science 316, 1905-1907 (2007).

35. Traulsen, A., Hauert, C., De Silva, H., Nowak, M. A. \& Sigmund, K. Exploration dynamics in evolutionary games. Proc. Natl Acad. Sci. USA 106, 709-712 (2009).

36. García, J. \& Traulsen, A. Leaving the loners alone: Evolution of cooperation in the presence of antisocial punishment. J. Theor. Biol. 307, 168-173 (2012).

37. Hauert, C., De Monte, S., Hofbauer, J. \& Sigmund, K. Volunteering as red queen mechanism for cooperation in public goods games. Science 296, 1129-1132 (2002).

38. Semmann, D., Krambeck, H.-J. \& Milinski, M. Volunteering leads to rock-paperscissors dynamics in a public goods game. Nature 425, 390-393 (2003).

39. Sasaki, T. \& Unemi, T. Replicator dynamics in public goods games with reward funds. J. Theor. Biol. 287, 109-114 (2011).

40. Sasaki, T. \& Uchida, S. Rewards and the evolution of cooperation in public good games. Biol. Lett. 10, 20130903 (2014).

41. Cressman, R., Song, J. W., Zhang, B. Y. \& Tao, Y. Cooperation and evolutionary dynamics in the public goods game with institutional incentives. J. Theor. Biol. 299, 144-151 (2012).

42. Sigmund, K., Hauert, C. \& Nowak, M. A. Reward and punishment. Proc. Natl Acad. Sci. USA 98, 10757-10762 (2001).

43. Sasaki, T., Brännström, A., Dieckmann, U. \& Sigmund, K. The take-it-or-leave-it option allows small penalties to overcome social dilemmas. Proc. Natl Acad. Sci. USA 109, 1165-1169 (2012).

44. Choi, J. K. \& Ahn, T. K. Strategic reward and altruistic punishment support cooperation in a public goods game experiment. J. Econ. Psychol. 35, 17-30 (2013).

45. Wu, J.-J., Li, C., Zhang, B.-Y., Cressman, R. \& Tao, Y. The role of institutional incentives and the exemplar in promoting cooperation. Sci. Rep. 4, 6421 (2014).
46. Rand, D. G., Dreber, A., Ellingsen, T., Fudenberg, D. \& Nowak, M. A. Positive interactions promote public cooperation. Science 325, 1272-1275 (2009).

47. Andreoni, J., Harbaugh, W. \& Vesterlund, L. The carrot or the stick: Rewards, punishments, and cooperation. Am. Econ. Rev. 93, 893-902 (2003).

48. Sefton, M., Shupp, R. \& Walker, J. M. The effect of rewards and sanctions in provision of public goods. Econ. Inquiry 45, 671-690 (2007).

49. Sutter, M., Haigner, S. \& Kocher, M. G. Choosing the carrot or the stick? Endogenous institutional choice in social dilemma situations. Rev. Econ. Stud. 77 1540-1566 (2010).

50. Hilbe, C. \& Sigmund, K. Incentives and opportunism: from the carrot to the stick. Proc. Bio. Sci. 277, 2427-2433 (2010).

51. Szolnoki, A. \& Perc, M. Correlation of positive and negative reciprocity fails to confer an evolutionary advantage: Phase transitions to elementary strategies. Phys. Rev. X 3, 041021 (2013)

52. Chen, X., Sasaki, T., Brännström, Å. \& Dieckmann, U. First carrot, then stick: how the adaptive hybridization of incentives promotes cooperation. J. R. Soc. Interface 12, 20140935 (2015).

53. Hauert, C. Replicator dynamics of reward \& reputation in public goods games. $J$ Theor. Biol. 267, 22-28 (2010).

54. Hofbauer, J. \& Sigmund, K. Evolutionary Games and Population Dynamics (Cambridge Univ Press, Cambridge, UK, 1998).

55. Cressman, R. \& Tao, Y. The replicator equation and other game dynamics. Proc. Natl Acad. Sci. USA 111, 10810-10817 (2014).

56. Blyth, C. R. On Simpson's paradox and the sure-thing principle. J. Am. Stat. Assoc. 67, 364-366 (1972).

57. Chuang, J. S., Rivoire, O. \& Leibler, S. Simpson's paradox in a synthetic microbial system. Science 323, 272-275 (2009).

58. Killingback, T., Bieri, J. \& Flatt, T. Evolution in group-structured populations can resolve the tragedy of the commons. Proc. Bio. Sci. 273, 1477-1481 (2006).

59. Kendal, J., Feldman, M. W. \& Aoki, K. Cultural coevolution of norm adoption and enforcement when punishers are rewarded or non-punishers are punished. Theor. Popul. Biol. 70, 10-25 (2006).

60. Casari, M. Emergence of endogenous legal institutions: Property rights and community governance in the Italian alps. J. Econ. Hist. 67, 191-226 (2007).

61. Horne, C. The enforcement of norms: Group cohesion and meta-norms. Soc Psychol. Q. 64, 253-266 (2001).

62. Kiyonari, T. \& Barclay, P. Cooperation in social dilemmas: free riding may be thwarted by second-order reward rather than by punishment. J. Pers. Soc. Psychol. 95, 826-842 (2008)

63. Chen, X., Gross, T. \& Dieckmann, U. Shared rewarding overcomes defection traps in generalized volunteer's dilemmas. J. Theor. Biol. 335, 13-21 (2013).

64. Boyd, R., Gintis, H., Bowles, S. \& Richerson, P. J. The evolution of altruistic punishment. Proc. Natl Acad. Sci. USA 100, 3531-3535 (2003)

65. Perc, M. Sustainable institutionalized punishment requires elimination of secondorder free-riders. Sci. Rep. 2, 344 (2012).

66. Sasaki, T., Uchida, S., Cang, V. \& Chen, X. Evolution of sanctioning systems and opting out of games of life. arXiv preprint, arXiv:1411.5215 (2014).

67. Gächter, S. Carrot or stick? Nature 483, 39-40 (2012).

68. Berlin, I. Four Essays on Liberty (Oxford Univ Press, London, UK, 1969).

\section{Acknowledgments}

We thank Boyu Zhang for comments and suggestions. We also thank the Conference "Evolution of Cooperation", 8-10 April 2014, Sino-German Center for Research Promotion, Beijing, China, sponsored by National Natural Science Foundation of China (NSFC) and International Institute for Applied Systems Analysis (IIASA). T.S. was supported by the Foundational Questions in Evolutionary Biology Fund: RFP-12-21 and the Austrian Science Fund (FWF): P27018-G11.

\section{Author contributions}

T.S., S.U. and X.C. designed and wrote the paper. T.S. and S.U. performed research

\section{Additional information}

Supplementary information accompanies this paper at http://www.nature.com/ scientificreports

Competing financial interests: The authors declare no competing financial interests. How to cite this article: Sasaki, T., Uchida, S. \& Chen, X. Voluntary rewards mediate the evolution of pool punishment for maintaining public goods in large populations. Sci. Rep. $\mathbf{5}$, 8917; DOI:10.1038/srep08917 (2015)

This work is licensed under a Creative Commons Attribution 4.0 International License. The images or other third party material in this article are included in the article's Creative Commons license, unless indicated otherwise in the credit line; if the material is not included under the Creative Commons license, users will need to obtain permission from the license holder in order to reproduce the material. To view a copy of this license, visit http://creativecommons.org/licenses/by/4.0/ 